\title{
UNA PROPUESTA PARA CONSIDERAR EL MODELO DE BLACK Y SCHOLES COMO UNA NUEVA MÉTRICA DE CREACIÓN DE VALOR EN LAS ORGANIZACIONES. INVESTIGACIÓN APLICADA A EMPRESAS QUE COTIZAN EN LA BOLSA MEXICANA DE VALORES
}

\author{
Juan Alberto Adam Siade \\ Hortensia Lacayo Ojeda \\ Alejandra Cabello Rosales \\ Profesores del programa de tutores del doctorado de la FCA-UNAM.
}

\begin{abstract}
RESUMEN
Este artículo es fruto de una investigación doctoral realizada en la Facultad de Contaduría y Administración de la Universidad Nacional Autónoma de México del segundo semestre de 2000 a 2005.

Tiene como propósito presentar los resultados de la investigación mencionada en lo que respecta a la aplicación del modelo de Black y Scholes en empresas mexicanas que cotizan en bolsa.

Si se aplicara el modelo Black y Scholes con el criterio de dividendos equivalente al rendimiento real de la tasa libre de riesgo, en un proceso de negociación, los empresarios y los ejecutivos contarían con una información adicional sobre el valor que la empresa podría generar en el futuro que se relaciona con el RION, el RSI y la UAIIDA.

Este modelo que con bases probabilísticas sólidas indica qué valor podría tener la empresa en el futuro, nos permitiría determinar si la empresa fue capaz de generar valor o no en relación con el valor que de ella misma se esperaba.

Palabras clave: Valuación de empresas, creación de valor, Black y Scholes, Bolsa Mexicana de Valores.
\end{abstract}

\section{INTRODUCCIÓN}

\section{Planteamiento}

Existen diversos métodos para asignarle valor a una empresa, así como diversas métricas para saber si la empresa genera valor económico o no. Dentro de los métodos de valuación de empresas en esta investigación se estudian los siguientes: el Método de valor en libros, el Método de valor de mercado, el Método goodwill, el Método de flujos de efectivo descontados, el Método del múltiplo precio-utilidad y el Modelo de Black y Scholes. Las métricas de creación de valor que se estudian son: el Valor Económico Agregado $\left(\mathrm{EVA}^{\mathrm{TM}}\right)^{1}$, la

1 El EVA es una marca registrada por Stern Stewart \& Co. En este trabajo, solo se estudia para cuestiones de investigaciónacadémica. 
Generación Económica Operativa (GEOß), el Rendimiento sobre la Inversión (RSI), el Rendimiento Sobre la Inversión Operativa Neta (RION®) ${ }^{2}$, la Utilidad Antes de Impuestos, Intereses, Depreciaciones y Amortizaciones (UAIIDA) y el rendimiento bursátil.

El estudio y la investigación con respecto al modelo de Black y Scholes se han desarrollado considerablemente desde que fue creado en 1973. En la valuación de empresas, este modelo ha cobrado auge, ya que presenta, con bases probabilísticas sólidas, el valor que puede tener una empresa en el futuro. Este modelo, que se utiliza para valuar una opción, es aplicable a la valuación de una empresa, porque calcular el precio de las acciones de un negocio es equivalente a obtener el valor de la opción de compra de las mismas. En la aplicación de la fórmula de Black y Scholes hay que considerar que el activo subyacente, sobre el cual funciona una opción puede ser entre otras cosas acciones, bonos, oro, materias primas, edificios, o la opción de adquirir otra opción.

Se busca saber de que forma el modelo de Black y Scholes se relaciona con los métodos y las métricas mencionadas para considerarlo, además, como una métrica de creación de valor en las empresas.

\section{Pregunta de investigación}

¿Cuáles de los métodos de valuación de empresas y cuáles de las métricas de creación de valor mencionadas tienen mayor consistencia con el modelo de Black y Scholes?

Para los efectos de esta investigación por consistencia entenderemos la relación lineal que existe entre el modelo de Black y Scholes con las variables de las dos categorias en estudio. Estas dos categorías son los métodos de valuación de empresas y las métricas de creación de valor, como se explica más adelante en el apartado de metodología. En este sentido, por consistencia también entenderemos la relación que existe cuando se observa una tendencia similar en la comparación que se hace entre estas dos categorias y el modelo mencionado.

\section{Objetivo de la Investigación}

Analizar cuáles de los métodos de valuación de empresas y cuáles de las métricas de creación de valor mencionadas tienen mayor consistencia con el modelo de Black y Scholes.

\section{Hipótesis}

Los métodos de valuación de empresas y las métricas de creación de valor que mayor consistencia tienen con el modelo de Black y Scholes son el Método de valor en libros, el Método goodwill, el RSI, el RION y la UAIIDA, que son los que tienen más elementos que los otros métodos analizados en esta investigación, para brindar información en cuanto a la capacidad que la empresa tiene para generar valor.

\section{Metodología}

Con base en el objetivo, esta investigación está manejando dos categorías de variables.

- Las que se consideran métodos de valuación de empresas, que son: Método de valor en libros, Método de valor de mercado, Método goodwill, Método de

2 GEO y RION son marcas registradas por la Sociedad Panamericana de Estudios Empresariales, A.C. En este trabajo, solo se estudian para cuestiones de investigación académica. 
flujos de efectivo descontados y Método del múltiplo precio-utilidad.

- Las que se consideran métricas de creación de valor que son: el EVA, la GEO, el RSI, el RION, la UAIIDA y el rendimiento bursátil.

Se obtuvo la información financiera de las empresas contenidas en las bases de datos de la Bolsa Mexicana de Valores y en los boletines trimestrales de la propia Bolsa.

La información se seleccionó y se clasificó de acuerdo a los requerimientos de cada métrica y método para su cálculo.

Para obtener el resultado de las métricas y las variables se manejaron aproximadamente 16800 datos.

Dado que se estudia el valor de las empresas y la creación de valor en el transcurso del tiempo, se analizaron los estados financieros a fechas de cierre en serie continua de los años (1992-1997), (19972002) y (1992-2002) de las empresas de los sectores de alimentos, bebidas y tabaco; comercio; comunicaciones; construcción; empresas controladoras; industria de la transformación; y otros servicios, que al inicio de esta investigación formaban parte de la muestra del Índice de Precios y Cotizaciones (IPC) de la Bolsa Mexicana de Valores que son comparables en todos los casos al existir y cotizar todas ellas en los años mencionados. Estas empresas, por emisora, son: ALFA, APASCO, BIMBO, CEMEX, COMERCI, CONTAL, DESC,
FEMSA, GCARSO, GISSA, ICA, KIMBER, PEÑOLES, SAVIA, SORIANA, TAMSA, TELMEX, TELEVISA, y WALMEX. ${ }^{3}$

Este trabajo se originó a finales de 2002 y se concluyó a finales de 2004 , por esta razón las últimas cifras de cierre disponibles para la investigación cuando comenzaron las labores de recopilación de la información fueron las de 2002.

Estas 19 empresas de la muestra del Índice de Precios y Cotizaciones son las que se pueden estudiar en todo el periodo. Además, representan el $30.64 \%$ de las empresas emisoras que actualmente operan que son $62^{4}$ de las $160^{3}$ registradas en la Bolsa Mexicana de Valores.

Con base en la información financiera de las empresas, se calcularon los valores de cada uno de los métodos de valuación y de las métricas de valor económico, para descubrir estadística y analíticamente la consistencia de ellos con el modelo de Black y Scholes.

Se hizo un análisis financiero comparativo de la relación que tienen estos valores entre sí para conocer su consistencia a través de correlaciones, variaciones, tendencias y porcentajes.

El análisis se realizó en diferentes aspectos:

- Análisis de los coeficientes de correlación

- Análisis gráfico de tendencias

- Análisis de la observación de resultados obtenidos en el cálculo de los métodos y métricas.

\footnotetext{
3 No se incluye el sector financiero por los cambios continuos que éste ha tenido en la última década, lo que hace imposible dar un seguimiento en las cifras de los estados financieros. Diversas instituciones que fueron adquiridas, o que se fusionaron con otras, cambiaron radicalmente, o incluso desaparecieron. Esto provoca que existan grupos financieros con información financiera muy reciente por su creciente creación y que sus cifras hayan cambiado drásticamente de un momento a otro. Todo esto provoca que sus características no sean constantes con las empresas que forman parte del estudio y, por lo tanto, su información no pueda ser comparable.

4 Bolsa Mexicana de Valores (BMV), «emisoras», México, www bmv.com .mx. (10 de junio de 2004).

5 Idem.
} 
Se considera que el análisis de los coeficientes de correlación es el más adecuado y principal para el objetivo de la investigación, dado que lo que se busca es la relación lineal entre las variables, para poder proponer un método que permita integrar a los estados financieros información sobre su valor económico y la capacidad para generarlo.

El tipo de estudio fue correlacional, porque se mide la relación existente entre las variables bajo estudio; y longitudinal, porque se estudia la evolución de las empresas con datos de 1987 a 2002.

Una vez que se determinó cuáles de los métodos de valuación y de las métricas mencionadas en las dos categorías son los más consistentes con el modelo de Black y Acholes, se realizó una propuesta para utilizar una nueva métrica de creación de valor en las organizaciones con base en este modelo.

\subsection{Criterios de aplicación que se} siguieron para el cálculo de los métodos de valuación de empresas y de las métricas de creación de valor considerados en esta investigación

En este apartado se presentan los criterios de aplicación que se consideraron para los efectos de esta investigación, tanto para el cálculo de los métodos de valuación de empresas como de las métricas de creación de valor. De igual forma, se presentan los criterios de análisis que se utilizaron en función de las características de la información que se estudia en cada método.

\section{A. Modelo Black y Scholes}

El valor obtenido con el modelo Black y Scholes se calculó con dos criterios: considerando el porcentaje real de los dividendos erogados en el periodo de estudio; y considerando una tasa constante en función a un dividendo equivalente al rendimiento real de la tasa libre de riesgo.

En todos los casos, para calcular el valor de la empresa se tomó el tiempo de cinco años con los siguientes criterios:

Para calcular el valor de la empresa a cierre de 1992, se consideró como valor del subyacente (que representa el activo actual) el activo total de 1987 de las empresas, actualizado por medio del Índice Nacional de Precios al Consumidor (INPC), al cierre de 1992; como valor de ejecución (que representa el pasivo futuro), se tomó el pasivo de las empresas en 1992, que en este caso es el que tuvo la empresa 5 años después de 1987, es decir, es el pasivo futuro. Con este criterio, se tomó el pasivo que realmente tuvo la empresa en el periodo de estudio, evitando las imperfecciones de las estimaciones que, difícilmente, se ajustan a la realidad. De igual forma, con este criterio se logra el objetivo de la investigación, porque permite que un modelo de valuación se analice con las cifras que realmente presenta la empresa y no con estimaciones que forzosamente tienen algo de subjetivo. Con esto, el análisis se realiza de la manera más objetiva posible al manejar cifras reales en todo momento ${ }^{6}$.

6 Se considera pertinente hacer esta aclaración de la subjetividad y la objetividad porque se ha observado que la aplicación de estos modelos muchas veces se basa en estimaciones del valor de los pasivos. En este caso las características del estudio permiten manejar cifras reales. Sin embargo, es necesario aclarar que si estos modelos se aplican para calcular el valor que la empresa tendrá en el futuro $(2007,2012,2014$, etc.), forzosamente se tendrá que basar en una estimación que tendrá que estar financieramente soportada y justificada. 
De esta misma manera, para calcular el valor de la empresa a cierre de 1997 , se consideró como valor del subyacente (que representa el activo actual) el activo total de 1992 de las empresas, actualizado por medio del INPC a cierre de 1997; como valor de ejecución (que representa el pasivo futuro), se tomó el pasivo de las empresas en 1997, que en este caso es el que tuvo la empresa 5 años después de 1992, es decir, es el pasivo futuro. Y el mismo criterio para el valor en el año 2002.

El criterio de análisis de Black y Scholes debe ser muy crítico, porque éste surge de un modelo probabilístico diseñado para valuar opciones de compra, es decir, de un modelo que nos indica cuánto valdrá una opción en el futuro. Por lo general, las opciones de compra se manejan por periodos muy cortos, es decir, de entre unos cuantos días hasta uno, dos o tres meses. Cuando el modelo lo trasladamos a valuar una empresa, no nos interesa saber cuanto valdrá dentro de 8 días o dentro de un mes y medio, sino que nos interesa saber cuál será su valor de compra dentro de 5 ó 10 años para saber si conviene invertir o no en ella.

Si se aplicara el modelo de Black y Scholes para valuar una empresa a un periodo de un mes nos llevaríamos la sorpresa de que su valor es en gran medida similar al del valor en libros, porque el subyacente no ha cambiado considerablemente y el pasivo futuro tampoco. Entonces, se deduce que no es un modelo que nos sea útil para valuar empresas en periodos tan pequeños, como lo puede ser para valuar una opción de compra de una acción en el mercado que de un día para otro sufre grandes alteraciones.

Con este razonamiento se puede deducir que, para los efectos de esta investigación, se calculó el valor que el modelo Black y Scholes le asignaba a las empresas en los años 1992, 1997 y 2002, considerando cifras reales para compararlo con los valores en libros que en realidad obtuvieron las mismas. Es decir, es un modelo que nos indica -en función del tiempo que puede ser desde un día hasta varios años- cuál será el valor de compra, en este caso de la empresa, en el futuro. En este sentido, el criterio de aplicación fue el de preguntarnos: ¿cuánto valdrá la empresa en 1992, 1997 y 2002 si calculamos su valor 5 años antes? Lo que permitió compararlo con el valor que la empresa tuvo en la realidad.

En todos los casos, como ya se ha mencionado, el tiempo fue de 5 años, la tasa de interés libre de riesgo fue la del promedio anual de los Certificados de la Tesorería de la Federación (Cetes) a 28 días y la volatilidad fue la que presentaron las empresas en los años del estudio. Como ya se mencionó, se tomaron dos criterios para el manejo de los dividendos, porque el modelo está diseñado para el cálculo del valor cuando el porcentaje de los dividendos es constante. Para manejarlo así, se promedió la tasa de dividendo real erogada por la empresa en el periodo de estudio, y, por otra parte, se consideró una tasa constante de dividendos en relación con la tasa libre de riesgo real del periodo de estudio.

Este trabajo aplica el modelo con la aportación de Merton en relación con los dividendos.

\section{B. Método de valor en libros}

El valor en libros es el monto del capital contable de las empresas en estudio y se tomó a la fecha de cierre de los años estudiados 1992, 1997 y 2002 . Al igual que todos los métodos, los resultados se 
manejaron con pesos actualizados a cierre del año 2002, actualizando las cifras con INPC.

\section{Método de valor de mercado}

Para calcular el valor de mercado se multiplicó el valor en libros de cada empresa por el múltiplo de precios / valor en libros (P/VL) que representa la relación que hay entre el valor en libros de las acciones de la empresa y el valor de las acciones en el mercado o valor de mercado de las acciones.

Otra forma de calcularlo es multiplicando el número de acciones por el valor de mercado de cada acción.

Las acciones representan la parte alícuota del capital de una empresa. Al multiplicar este capital o valor en libros por el múltiplo que relaciona el valor de mercado de la acción con el valor en libros de la misma, se obtiene, en consecuencia, el valor de mercado de la empresa ${ }^{7}$.

Cuando el valor del múltiplo es superior a uno (01), significa que el valor de mercado está arriba del valor en libros; $y$, por el contrario y lógicamente, cuando el valor del múltiplo es inferior a uno (01), significa que el valor de mercado está por abajo del valor en libros.

\section{Método goodwill}

El valor obtenido por el Método goodwill se calculó de dos formas: La manera tradicional, comparando la utilidad neta ponderada de los últimos cinco años con el rendimiento mínimo esperado por los inversionistas; y comparando el rendimiento operativo ponderado de los últimos cinco años con el rendimiento mínimo esperado por los inversionistas. Es decir, para obtener el valor de la empresa por el Método goodwill tradicional se calcula el rendimiento neto de la empresa de los últimos cinco años. Para los efectos de esta investigación el goodwill se calculó, además, con el resultado operativo de las empresas, para intentar relacionarlo más con las métricas de creación de valor que consideran el propio resultado operativo para su cálculo. Con esto se manejan dos nombres el goodwill neto (tradicional) y el operativo.

Para calcular el goodwill operativo ${ }^{8}$, el resultado de operación de los últimos cinco años se actualiza a pesos del año más actual, es decir, del último año. Posteriormente, se suman dichos rendimientos y se ponderan en partes iguales, es decir, al $20 \%$ o, lo que es lo mismo, entre cinco años. Ese rendimiento se compara con el rendimiento mínimo que los inversionistas esperan recibir por su inversión. Si el rendimiento obtenido es mayor que el esperado, la empresa está generando valor económico reflejado en un goodwill operativo positivo; pero, si es inferior al esperado por los inversionistas, está destruyendo valor económico reflejado en el badwill o goodwill operativo negativo. Posteriormente, al resultado se le suma los activos netos de la empresa para obtener el valor de la misma en función del Método goodwill.

Dadas las características del Método goodwill, es el único que se puede correlacionar de dos formas con las métricas de creación de valor, porque en su cálculo se obtienen dos resultados.

7 Con base en: DÍAZ MATA, Alfredo. Invierta en la bolsa.p. 233.

8 Se siguen los mismos pasos que en el goodurilltradicional, pero con la diferencia de que se considera el resultado operativo y no el resultado neto 


\section{E. Método de flujos de efectivo descontados}

En una investigación de campo previa, fueron entrevistados diversos empresarios y ejecutivos en relación con los métodos de valuación de empresas. En ella se mencionó que el problema del método de flujos de efectivo descontados, que es el que más se usa para valuar una empresa, es que las proyecciones se realizan con criterios subjetivos que nada tienen que ver con la realidad de lo que la empresa será capaz de generar, o sea, que se crean con cifras que se presentan dependiendo del lado de la mesa en el que se esté, es decir, una cifra si se es el vendedor y otra si se es el comprador'.

Para el cálculo del valor de la empresa con el método de flujos de efectivo descontados se consideró el valor del incremento o decremento neto en el efectivo de las empresas, con lo que se cuidaron los detalles y criterios de objetividad que se deben tener por el problema que se presenta al hacer una proyección de los propios flujos. Para los efectos de esta investigación el problema de las proyecciones se subsanó por la misma naturaleza de la investigación, que al manejar flujos descontados por cinco años para calcular el valor de las empresas, se tomaron los flujos reales generados por las mismas. Con esto se siguió el mismo criterio de objetividad tomado para el cálculo del modelo Black y Scholes, es decir, se tomaron cifras reales de los flujos de efectivo que generó la empresa.

Este es el motivo por el cual en los resultados de la investigación observamos que se presentaron valores negativos para la empresa al aplicar este método de valuación. Para calcular el valor de la empresa en 1992, se tomaron los flujos que en verdad la empresa generó de 1993 a 1997; para el cálculo del valor de la empresa en 1997, se tomaron en cuenta los flujos de efectivo que la empresa efectivamente generó de 1998 a $2002^{10}$. Al tomar los flujos que la empresa generó se observó que muchos de ellos fueron negativos, es decir, que en lugar de haber un incremento en el efectivo, se generó un decremento en el mismo, lo que provocó que, en sentido estricto de aplicación, las empresas tuvieran un valor negativo calculado con este método.

Cuando en una empresa se hace una proyección de flujos por cinco años, difícilmente se pueden proyectar cifras negativas si se es parte de la administración que está en la postura del vendedor, pero si se es el comprador se analizarán los flujos pasados para ver si se generó o no flujo positivo.

Los flujos que se consideraron en este trabajo fueron los recursos generados (utilizados) por la operación, por el financiamiento y por la inversión, con lo que se obtuvo el incremento o el decremento neto en efectivo ${ }^{11}$. Esta información es la que se obtiene del Estado de Cambios en la Situación Financiera.

La investigación realizada, al tomar las cifras que realmente se presentaron en las empresas, elimina el elemento subjetivo, lo

- Conbase en:URIARTE, Héctor M. (coordinador) Valuación de empresas y creación de valor. Cap. 5, pp. 117-256. Cabe aclararque para el cálculo del valor de las empresas por este método para el año 2002, al no tener los datos de cierre de 2003 en adelante, al momento de realizar este estudio porque éstos todavía no ocurrían al momento de hacer el cálculo, se tomaron las tendencias de los 10 años pasados en la generación de los flujos, para evitar caer en una subjetividad de proyecciones a futuro que no irían con la naturaleza de la investigación que se desarrolló.

Este criterio de valuación es recomendado por VILLANUEVA, C. Siu. Valuación de empresas, pp. 67-228. 
que permite analizar los resultados y compararlos en la forma pura y objetiva que permite sacar conclusiones más apegadas a la realidad de cada método y modelo.

Cabe aclarar que hay otras formas de aplicar este método en función de los flujos libres de efectivo como lo mencionan Sanjurjo y Reinoso ${ }^{12}$, quienes señalan que al resultado, antes de intereses e impuestos, hay que aumentarle los impuestos, restarle las amortizaciones, sumarle o restarle las variaciones en el capital de trabajo y sumarle o restarle las inversiones netas, para obtener un flujo libre de efectivo. Sin embargo, esta alternativa tampoco nos garantiza que no se han de presentar flujos negativos.

Para los efectos de esta investigación se consideró más pertinente el criterio de flujo en relación con el incremento o decremento neto de efectivo, que el de flujo libre de efectivo, porque este último no considera los flujos financieros de la empresa, que para los efectos de valuación son muy importantes.

Aun si al flujo libre de efectivo se le aumentaran o disminuyeran las variaciones de inversiones financieras temporales, las variaciones en el endeudamiento financiero, las reducciones o incrementos del capital social, los pagos y cobros de intereses; y se le sumaran los pagos de dividendos para obtener un flujo de efectivo financiero, se considera que el criterio de incremento o decremento neto de efectivo estudiado en este trabajo es el más representativo para los efectos de valuación como lo recomienda Siu Villanueva, al calcular tanto el valor de los flujos de efectivo descontados como del negocio en marcha.

Además, se considera que para lo que se busca en esta investigación que es relacionar métodos de valuación con métricas de generación de valor, con este criterio se está obteniendo un valor más adecuado para la empresa que el que se pudiera obtener con ajustes a los propios flujos.

\section{F. Método del múltiplo precio-utilidad}

El valor obtenido por el método del múltiplo precio-utilidad se calculó de la manera tradicional, multiplicando la utilidad neta ponderada de los últimos cinco años por el múltiplo precio-utilidad presentado por cada empresa en los años de estudio.

\section{G. EVA (Valor Económico Agregado) y GEO (Generación Económica Ope- rativa)}

El EVA (Valor Económico Agregado) y la GEO (Generación Económica Ope-rativa) se obtuvieron con dos criterios para el cálculo del costo de capital: el criterio tradicional con el modelo Capital Asset Priang Model (CAPM) y el criterio de costo de capital propio en relación con la tasa libre de riesgo. De ambos criterios se considera que el que mayor soporte financiero tiene es el CAPM ${ }^{13}$.

SANJURJO, Miguel y REINOSO, María. (coordinadores) Guia de valoración de empresas. p. 220

$\mathrm{S}_{1}$ bien el modelo CAPM tiene algunas desventajas para ser aplicado al caso mexicano, porque en ocasiones el rendimınto del mercado es negativo, es decir, reporta pérdidas, esto mismo hace que se considere importante como valor de referencra para las empresas que pertenecen a un país cuyo mercado reporta pérdıdas y, por lo tanto. sus niveles minımos de rendimiento, también se ven afectados por el mismo mercado. Para los efectos de esta ınvestıgacion se consıdera importante observar los valores de referencia de ambos criterios de aplicación. Además es importante consıderar que si las características económicas de un país provocan que el mercado presente pérdidas o cifras negatıvas, ¿en que medida se les puede exigir a las empresas costos de capital elevados?, es decir.el hecho de que el mercado presente cifras negatıvas es un elemento importante a considerar en la economía, que en función del modelo es un hecho que se tiene que ver reflejado en el costo de capital de las empresas. 


\section{H. RION, RSI, UAIIDA y rendimiento bursátil}

Para el cálculo de estas métricas se tomaron los datos propios de los estados financieros y los obtenidos en los anuarios bursátiles y financieros de la Bolsa Mexicana de Valores.

\section{Resultados}

Con base en las características observadas del comportamiento del modelo de Black y Acholes, calculado con el criterio de dividendos equivalente al rendimiento real de la tasa libre de riesgo, se puede afirmar que, aunque el valor que arroja el modelo no es el valor que la empresa tendrá en el futuro, sí puede ser considerado como elemento de referencia, en cuanto al valor que de la empresa se espera a uno o más años.

En este sentido, si el valor en libros que la empresa obtenga en el futuro (a un año) es inferior al valor esperado, la organización no generó el valor que como mínimo se esperaba obtener, o, por el contrario, si el valor en libros que la empresa obtenga en el futuro es superior al valor esperado, quiere decir que generó cierto valor que rebasó las expectativas.

También se puede afirmar que el modelo de Black y Scholes, calculado con el criterio mencionado, es más acertado que el método de flujos de efectivo descontados aplicado con los criterios ya señalados. Estos métodos son comparables porque ambos son los únicos que se adelantan a lo que la empresa valdrá en el futuro; el modelo de Black y Scholes con bases probabilísticas sólidas y el de flujos de efectivo con bases en proyecciones.

Para los efectos de esta investigación el problema de las proyecciones se subsanó por la misma naturaleza de la investigación que al manejar flujos descontados por cinco años para calcular el valor de las empresas, se tomaron los flujos reales generados por las mismas. Por esto se observa que hay valores negativos para la empresa al aplicar este método de valuación.

Esta investigación, al tomar las cifras que realmente se presentaron en las empresas, elimina el elemento subjetivo, lo que permite analizar los resultados y compararlos en la forma pura y objetiva que permite sacar conclusiones más apegadas a la realidad de cada método y modelo.

Por otro lado, en el análisis de los coeficientes de correlación también se observa que el modelo de Black y Acholes, con el criterio de dividendos equivalentes al rendimiento real de la tasa libre de riesgo, presenta correlaciones importantes en relación con las métricas presentadas en pesos RION, RSI y UAIIDA (Cuadro N. ${ }^{\circ} 1$ ), y no así en todos los casos con las métricas EVA, GEO y rendimiento bursátil (Cuadro N. ${ }^{\circ}$ ). Se observa una relación importante con los métodos de valor en libros, goodwill, múltiplo precio-utilidad y valor de mercado (Cuadro N. ${ }^{\circ} 3$ ). Cabe destacar que con el que más se relaciona es con el goodwill. 
Cuadro N. ${ }^{\circ} 1$

Correlación entre el modelo Black y Scholes con el criterio de dividendo equivalente al rendimiento real de la tasa libre de riesgo, y las métricas de creación de valor RION, RSI y UAIIDA

\begin{tabular}{lccc}
\hline & 1992 & 1997 & 2002 \\
\hline & BySDIVTL & BySDIVTL & BySDIVTL \\
RION & 0.748 & 0.977 & 0.875 \\
RSI & 0.718 & 0.669 & 0.8 \\
UAIIDA & 0.753 & 0.981 & 0.877 \\
\hline
\end{tabular}

Fuente: Elaboración propia.

Cuadro N. ${ }^{\circ} 2$

Correlación entre el modelo Blacky Scholes con el criterio de dividendo equivalente al rendimiento real de la tasa libre de riesgo, y las métricas de creación de valor EVA, GEO y rendimiento bursátil

\begin{tabular}{llll}
\hline & \multicolumn{1}{c}{1992} & 1997 & \multicolumn{1}{c}{$\mathbf{2 0 0 2}$} \\
\hline & BySDIVTL & BySDIVTL & BySDIVTL \\
EVACAPM & 0.510 & -0.890 & 0.915 \\
GEOCAPM & -0.587 & -0.952 & 0.855 \\
RENDBUR & -0.285 & 0.153 & -0.113 \\
\hline
\end{tabular}

Fuente: Elaboración propia.

CuadroN. ${ }^{\circ} 3$

Correlación entre el modelo Black y Scholes con el criterio de dividendo equivalente al rendimiento real de la tasa libre de riesgo, y los métodos devaluación de empresas considerados en el estudio

\begin{tabular}{llll}
\hline & 1992 & 1997 & 2002 \\
\hline VLIBROS & BySDIVTL & BySDIVTL & BySDIVTL \\
GOODWILL & 0.794 & 0.969 & 0.908 \\
MULT.PU & 0.694 & 0.98 & 0.928 \\
V MERCADO & 0.654 & 0.912 & 0.84 \\
FLUJOSDC & 0.388 & 0.922 & 0.85 \\
\hline
\end{tabular}

Fuente: Elaboración propia. 
En el análisis comparativo en el mismo modelo Black y Scholes con el criterio de porcentaje real de los dividendos erogados, es decir, con el otro criterio de aplicación, se observa en los cuadros 4 y 5 que en relación con las mismas variables, también existen correlaciones positivas, pero en un nivel considerablemente inferior a las obtenidas con el otro criterio.

\section{Cuadro N. 4}

Correlación entre el modelo Black y Scholes con el criterio de porcentaje real de los dividendos erogados, y las métricas de creación de valor RION, RSI y UAIIDA

\begin{tabular}{|l|r|r|r|}
\hline & \multicolumn{1}{|c|}{1992} & \multicolumn{1}{c|}{1997} & \multicolumn{1}{c|}{2002} \\
\hline & BySDIVPA & BySDIVPA & BySDIVPA \\
\hline RION & 0.444 & 0.684 & 0.415 \\
\hline RSI & 0.428 & 0.642 & 0.299 \\
\hline UAIIDA & 0.448 & 0.662 & 0.409 \\
\hline
\end{tabular}

Fuente: Elaboración propia.

CuadroN. ${ }^{\circ} 5$

Correlación entre el modelo Black y Scholes con el criterio de porcentaje real de los dividendos erogados, y los métodos de valuación de empresas considerados en el estudio

\begin{tabular}{|l|r|r|r|}
\hline & \multicolumn{1}{|c|}{1992} & \multicolumn{1}{c|}{1997} & \multicolumn{1}{c|}{2002} \\
\hline & BySDIVPA & BySDIVPA & BySDIVPA \\
\hline VLIBROS & 0.531 & 0.702 & 0.655 \\
\hline GOODWILL & 0.547 & 0.67 & 0.619 \\
\hline MULT.PU & 0.381 & 0.519 & 0.339 \\
\hline VMERCADO & 0.412 & 0.651 & 0.446 \\
\hline FLUJOSDC & 0.171 & 0.367 & 0.045 \\
\hline
\end{tabular}

Fuente: Elaboración propia.

6. Propuesta para considerar el modelo Black y Scholes como una métrica de creación de valor

El modelo Black y Scholes puede onndar una información adicional a la encontrada en los estados financieros de las rrganizaciones que se relaciona con lo que bempresa puede generar del valor financiero redeiado en el RION, el RSI y al UAIIDA. Jomo se señaló con anterioridad, este - odelo no nos va a dar el valor exacto de la -mpresa en el futuro, sino que, dadas las zaracterísticas del mismo, que con bases probabilísticas sólidas indica el valor que en el futuro podría tener la empresa, nos señalaría si la empresa fue capaz de generar valor o no.

Si la empresa por medio del modelo Black y Scholes calcula un valor a un año y transcurrido este tiempo se observa que el valor en libros fue superior, entonces, se puede concluir que la empresa generó valor financiero, ya que superó las expectativas probabilísticas que se esperaban. Por el contrario, si se observa que la empresa reporta un valor en libros menor al que las 
expectativas probabilísticas del modelo nos indicaban, entonces se puede decir que la empresa no alcanzó el valor mínimo que se esperaba de ella en función de las bases probabilísticas del modelo, lo que indicaría que la empresa no generó valor financiero que, para este caso, no es el que miden las métricas conocidas, sino que sería un valor en relación con el modelo aplicado, que podría ser llamado «valor económico futuro minimo esperados.

En el estudio se observa cómo el valor que arroja este modelo en algunas ocasiones rebasa el valor en libros y en otras tantas se queda por debajo, coincidiendo en el $68 \%$ de las ocasiones con el aumento o disminución de las métricas de creación de valor EVA o con las del goodwill.

Se considera que se puede tomar como referencia el valor en libros, porque el propio modelo de Black y Scholes toma las variables que se estudian en el valor en libros que son los activos como valor del subyacente y los pasivos como valor de ejercicio en la fórmula, así como los dividendos y las variables que hacen del modelo uno de los más acertados para valuar opciones.

6.1. Ejemplo del uso del modelo Black y Scholes como métrica de creación de valor

\section{NOMBRE DE LA EMPRESA}

\section{Reporte de generación de valor al 31 de diciembre de 2005}

Valor en libros ${ }^{(1)}$

$\$ 6000000.00$

Valor minımo esperado al cierre del presente año $2005^{(2)}$

6100000.00

Valor mínimo esperado al cierre del próximo año $2006^{(3)}$

6160000.00

Valor en libros superior o inferior al mínimoesperado al cierre del presente año(4)

$(100000.00)$

(1) Valor delcapital contable del año.

(2) Valor calculado el cierre del año anterior 2004, delo que se espera a cierre de 2005 con el modelo de Black y Scholes Schole .alculado al cierre del presente año 2005, de lo que se espera para el cierre de 2006 con el modelo de Black y

(4) Valor superior o inferior al proyectado por el modelo de Blacky Scholes, considerando dividendos. Es la diferencia entre el valor mínımo esperado al cierre del presente año (punto 2), y el valor en libros (punto 1).

En este caso, al cierre de 2005, la empresa había generado $\$ 100000.00$ menos de lo esperado, es decir, se esperaba que tuviera un valor en libros de $\$ 6100000.00$; y su valor al final del año fue de $\$ 6000000$.00. Por ser negativo, aparece entre paréntesis

En el siguiente reporte, a cierre de 2005, se observa que el valor mínimo esperado para el presente año (punto 2) se calculó y registró en el estado financiero de 2006 (punto 3). Como se explicó anteriormente, es un valor proyectado de lo que la empresa podría valer en función del sustento probabilístico que tiene el modelo de Black y Scholes, considerando dividendos aplicados en esta investigación. Si el valor en libros es menor, entonces se dice que la empresa tiene un valor inferior al mínimo 
esperado; pero si es mayor, se dice que tiene un valor superior al mínimo esperado (punto 4). Este valor podría ser llamado como Valor Financiero Mínimo Esperado como propuesta de esta investigación.
Con estas señales los usuarios de la información tendrán más y mejores elementos en relación con el valor de la empresa en relación con su capacidad para generar valor.

\section{NOMBRE DE LA EMPRESA}

\section{Reporte de generación de valor al 31 de diciembre de 2006}

Valor en libros ${ }^{(t)}$

Valor mínimo esperado al cierre del presente año ${ }^{(2)}$

Valor mínimo esperado al cierre del próximo año ${ }^{(3)}$

Valor en libros superior o inferior al mínimoesperado

al cierre del presente año(4)
$\$ 6200000.00$

6160000.00

6450000.00

40000.00

Valor del capital contable del año.

(2) Valor calculadoel cierre del año anterior 2005, de lo que se espera a cierre de 2006, según el modelode Blacky Scholes.

(3) Valor calculado al cierre del presente año 2006, de lo que se espera para el cierre de 2007, según el modelo de Black y Scholes.

(4) Valor superior o inferior al proyectado por el modelo de Blacky Scholes, considerando dividendos. Es la diferencia entre el valor mínimo esperado al cierre del presente año (punto 2), y el valor en libros (punto 1).

\section{CONCLUSIONES}

$\mathrm{Al}$ comprobarse la hipótesis de que los métodos de valuación de empresas y las métricas de creación de valor que mayor consistencia tienen con el modelo de Black y Scholes son el Método de valor en libros, el Método goodwıll, el RSI, el RION yla UAIIDA, se puede asegurar que incluir este modelo en la información de las empresas permitirá utilizarlo como métrica de creación de valor.

En una valuación de empresas es tan importante considerar la historia de la organización como su posible futuro. Si se aplica el modelo Black y Scholes con el criterio de dividendos equivalente al rendimiento real de la tasa libre de riesgo, en un proceso de negociación, los empresarios $\checkmark$ los ejecutivos contarían con una información adicional sobre el valor que la empresa podría generar en el futuro que se relaciona con el RION, al RSI y al UAIIDA.

Este método Black y Scholes no nos va a brindar el valor exacto que la empresa tendrá en el futuro, sino que, dadas las características del mismo que con bases probabilísticas sólidas indica el valor que en el futuro podría tener la empresa, nos señalaría si la empresa fue capaz de generar valor o no en relación con el valor que de ella misma se esperaba.

Si el modelo de Black y Scholes, calculado con el criterio mencionado, determina el valor que una empresa tendrá en un año, y se observa que después de ese año el valor en libros rebasó la expectativa, es decir, que fue superior, se puede concluir que la empresa generó valor. Por el contrario, si el valor en libros no alcanzó las 
expectativas, es decir, que se quedó por debajo del valor pronosticado, se podría concluir que la empresa no generó valor. En este caso no sería un valor relacionado con las métricas conocidas, sino que sería una forma más como la empresa puede medir la creación de valor. En este caso podría llamarse valor económico futuro mínimo esperado.

El valor que calcula este modelo en algunas ocasiones rebasa el valor en libros $y$, en otras tantas, se queda por debajo, coincidiendo en casi el $70 \%$ de las ocasiones con el aumento odisminución de las métricas de creación de valor EVA o con las del goodwnll, porque es otro valor el que mide.

Se puede tomar como referencia el valor en libros, porque el propio modelo de Black y Scholes toma las variables que se estudian en el valor en libros, que son los activos, como valor del subyacente y los pasivos, como valor de ejercicio en la fórmula.

\section{REFERENCIAS}

ADAM SIADE, Juan Alberto. Análisis de los métodos de valuación de empresas y la relación de éstos con la capacidad que las organizaciones thenen para generar valor económico: propuesta para reportar, en la información financiera, el valor de las organizaciones y su capacidad para generarlo. México, Universidad Nacional Autónoma de México, Facultad de Contaduría y Administración, División de Estudios de Postgrado, investigación doctoral, 2005, 243 pp.

ALVARADO MARTINEZ DE ESCOBAR, Lourdes. La contaduría pública. México, UNAM, 1983, 144 pp.

AMERICANSOCIETY OF APPRAISERS. Business valuation: introduction to business valuation. Parte I, v. 1.0s (3/98), EE.UU., American Society of Appraisers, 1998.
BENNINGA, Simon Z.y Oded H. SARIG. Corporate finance a valuation approach. EEUU, McGraw-Hill, 1997, 445 pp.

BESLEY, Scott y BRIGHAM, Eugene F. Fundamentos de administración financiera. México, McGraw-Hill, 12. ${ }^{a}$ ed., 2001, 919 pp.

BLACK, Andrew, WRIGHT, Phillip y John DAVIS. In search of shareholder value. Prentice, 2001, 2. ${ }^{2}$ ed., 306 pp.

BLACK, Fisher y Miron SCHOLES. «The valuation of option contracts and a test of market efficiency». En: Journal of Finance 27, EE.UU., 1972, pp. 399-417.

BLACK, Fischer y Miron SCHOLES. «The pricing of options and corporate liabilities». En: Journal of Political Economy. Chicago, Universidad de Chicago, 1973, Vol. 81, N. ${ }^{\circ} 3$, mayo/junio.

BOLSA MEXICANA DE VALORES. «Anuarios financiero». 1987 - 2002. «Anuarios bursátiles». 1987-2002.

BREALEY, Richard A., y MYERS, Stewart C. Pincipios de finanzas corporativas. Madrid, McGraw-Hill, 1996, 4. ${ }^{2}$ ed., 1203 Pp.

CABELLO ROSALES, Alejandra. El modelo Black y Scholes y sus modificaciones. México, notas en preparación 2004.

DAMODARAN, Aswath. Damodaran on valuation: security analysis for investment and corporate finance. EE.UU., John Wiley \& Sons, 1994, 426 pp.

DAMODARAN, Aswath. Investment valuation. EE.UU., John Wiley \& Sons, 1996.

DAY, Alastair L. Mastering financial modeling. Londres, Financial Times Prentice Hall, Pearson Education, 2001, 370 pp. 
ECCLES, Robert G., HERZ, Robert H., KEEGAN, E. Mary y David M. H. PHILLIPS. The value reporting revolution. Nueva York, PricewaterhouseCoopers, 2001, 349. pp.

DIAZ MATA, Alfredo. Invierta en la bolsa. México, Grupo Editorial Ibérica, 1994, 2. ${ }^{\mathrm{a}}$ ed., $307 \mathrm{pp}$.

DIEZ DE CASTRO, Luis y MASCAREÑAS PÉREZ-ÍÑIGO, Juan. Ingeniería financiera. Madrid, Mc.Graw-Hill, 1994, 2. ${ }^{a}$ ed., 467 pp.

EVANS, Frank, C., y David M. BISHOP. Valuation for M $\mathcal{A} A$ : building value in private companies. EE.UU., John Wiley \& Sons, 2001, 298 pp.

FERRATER MORA, José. Diccionario de filosofía. Barcelona, Ariel, 2001, 4 tomos, $1^{\text {sa }}$ reimp., $3830 \mathrm{pp}$.

FISHMAN, Jay E., PRATT, Shannon P., GRIFFITH, J. Clifford, y D. Keith WILSON. Guide to business valuations. Texas, Practitioners Publishing Company, 1997, Vol. 1, 7. ed., Forth Worth.

HELFERT, Erich. Valuación. México, ECASA, 1981, 1. ${ }^{2}$ reimp., 167 pp.

IBBOTSON ASSOCIATES. Stocks, bonds, bills and inflation: sbbi valuation edition 1999 yearbook. Chicago, Illinois, Ibbotson Associates, 1999, 272 pp.

INSTITUTO MEXICANO DE CONTADORES PÚBLICOS $y$ ANFECA. Principios de contabilidad generalmente aceptados. México, IMCP, 2003, 18. ${ }^{2}$ ed., 678 pp.

INTERNATIONAL ACCOUNTING STANDARDS BOARD E INSTITUTO MEXICANO DE CONTADORES
PÚBLICOS. Normas internacionales de información financiera. México, IASB, IMCP, 2003, s/p.

KOHLER, Heinz. Estadística para negocios y economia. México, CECSA, 1999, 2. reimp. en español, 1053 pp.

MC TAGGART, James M., KONTES, Peter W, y MANKINS, Michael C. The value imperative: managing for superior. shareholders. EEUU, The free press, 1994, 367 pp.

MEIGS, Robert F, et al. Contabilidad. La base para decisiones gerenciales. Santafé de Bogotá McGraw-Hill, 1999, 881 pp., XIII pp. apéndice.

MORENO FERNÁNDEZ, Joaquín. Las finanzas en la empresa. México, Instituto Mexicano de Contadores Públicos, 1994, 5. ${ }^{\mathrm{e}}$ ed., $580 \mathrm{pP}$.

OCHOA TORRES, Miguel, QUIROZ ABED, Guillermo y V.ELARDE DABROWSKI, Raúl, Rion, geo y valore, tres instrumentos para generar valor, México, IPADE, 1997, $110 \mathrm{pp}$.

PRATT, Shannon P. Cost of capital: estimation and applications. EEUU, John Wiley \& Sons Inc., 1998, 226 pp.

PRATT, Shannon P.,REILLY, Robert F. y Robert P. SCHWEIHS. Valuing a business: the analysis and appraisal of closely beld companies. EEUU, Richard D. Irwin, 1996, 3. ${ }^{\mathrm{a}}$ ed., $850 \mathrm{pp}$.

RAPPAPORT, Alfred. Creating shareholder value, the new standard for business performance. Free press, 1986.

READ, Nick, y Jonathan BATSON. Spreadsheet modelling best practice. Londres, Business Dynamics and Price waterhouse Coopers, 1999, 204 pp. 
REAL ACADEMIA ESPAÑOLA. Diccionario de la lengua española,

RODRÍGUEZ DE CASTRO, J. Introducción al análisis de productos financieros derivados. México, Limusa, 1995, 258 pp.

ROSS, Stephen A., WESTERFIELD, Randolph W y JAFFE, Jeffrey F. Finanzas corporativas. México, Irvin McGraw-Hill, 2000, 5. ${ }^{2}$ ed., 1053 pp.

SAAVEDRA GARCÍA, María Luisa. La valuación de empresas. Enfoques teóricos y aplicación de los modelos Black y Scholes, valor económico agregado, y flujo de efectivo disponible en México: 1991-2000. México, Universidad Nacional Autónoma de México, Facultad de Contaduría y Adminis-tración, División de Estudios de Postgrado, Investigación doctoral, 2002, 175 pp.

SAUNDERS, Anthony. Financial institutions management. 3. ${ }^{\mathrm{ra}}$ ed., Irwin McGraw-Hill, EE.UU., 1999.

SHETH, Jagdish, y Rajendra SISODIA. The rule of three, surviving and thriving in competitive markets. Free Press, 2002.
SIU VILLANUEVA, Carlos, Valuación de empresas. México, IMCP, 2001, 230 pp.

SMITH, Gordon V. Trademark valuation. EE.UU., John Wiley \& Sons, 1997, 293 pp.

SOLNIK, Bruno. Inversiones internacionales. EE.UU., Adisson-Wesley Iberoamericana, 1991, $457 \mathrm{pp}$.

STERN, Joel M. Y John S. Shiely. The EVA. challenge, implementing value-added change in an organization. Wiley, 2001.

STEWART, III, G. Bennett. The questforvalue. EE.UU., Harper Business, 1991, 781 pp.

THE APPRAISAL FOUNDATION. Uniform Standards of Professional Appraisal Practice: 1999 Edition. EE.UU., The Appraisal Foundation, 1999, 164 pp.

URIARTE, Héctor M. (coordinador) Valuación de empresas y creación de valor. México, UNAM, PricewaterhouseCoopers, IMEF, 2002, 270 pp.

VAN HORNE, James C. Administración financiera. México, Prentice Hall, 9. ${ }^{\mathrm{a}}$ ed., 1993. 